\title{
Prenatal and postnatal administration of Lactobacillus GG reduced the occurrence of atopic disease in offspring
}

\author{
Kalliomäki M, Salminen S, Arvilommi H, et al. Probiotics in primary prevention of atopic disease: a randomised \\ placebo-controlled trial. Lancet 2001 Apr 7;357:1076-9.
QUESTION: In offspring at risk for atopic disease, does oral administration of Lactobacillus GG (LGG), given prenatally to mothers and postnatally to their infants, prevent atopic eczema?

Design

Randomised (unclear allocation concealment*), blinded (clinicians, patients, and outcome assessors),* controlled trial with 2 years of follow up.

\section{Setting}

Antenatal clinics in Turku, Finland.

\section{Patients}

159 pregnant women who had atopic disease, or $\geqslant 1$ first-degree relative (mother, father, or older sibling), or partner with atopic eczema, allergic rhinitis, or asthma. 132 women (83\%) and 132 children completed follow up.

\section{Intervention}

Mothers were allocated to LGG $\left(1 \times 10^{10}\right.$ colonyforming units [Valio Limited, Helsinki, Finland]) $(\mathrm{n}=77)$ or to placebo (microcrystalline cellulose) $(\mathrm{n}=82)$. The capsules were taken daily for 2 to 4 weeks before expected delivery. After delivery, breastfeeding mothers continued to take the capsules, or the children received the contents mixed with water by spoon. The capsules were taken postnatally for 6 months.

\section{Main outcome measures}

A diagnosis of atopic eczema, the main outcome measure, required pruritis or facial or extensor involvement, or both, and a chronic relapsing course (eczema for $\geqslant 1$ mo at the 24 mo study visit and on $\geqslant 1$ previous visit).

Finnish Foundation Paediatric Research

National Technology Agency of Finland;

Allergy Research

Foundation in

southwest Finland.

For correspondence

Dr $M$ Kalliomäki,

Department of

Paediatrics, Turku

University Hospital, P.O. Box 52, FI-20521

Turku, Finland.

\section{Main results}

Atopic eczema occurred in fewer children who had received probiotics than in those who received placebo $(\mathrm{p}=0.008)$ (table).

\section{Conclusion}

In offspring at risk for atopic disease, Lactobacillus GG given prenatally and postnatally reduced the occurrence of atopic eczema up to 2 years of age.

*See glossary.

Lactobacillus $G G v$ placebo for prevention of atopic disease in children up to 2 years of age $\dagger$

\begin{tabular}{lllll} 
Outcome & Lactobacillus GG & Placebo & RRR (95\% CI) & NNT (CI) \\
Atopic eczema & $23 \%$ & $46 \%$ & $49 \%(16$ to 68$)$ & 5 (3 to 16) \\
\hline tAbbreviations defined in glossary & &
\end{tabular}

\section{COMMENTARY}

The study by Kalliomäki et al found an impressive halving of the risk for atopic eczema at 2 years in those given probiotics-cultures of potentially beneficial bacteria of the healthy gut microflora. 2 questions need to be answered: are the results valid, and, if so, should clinicians modify their practice now?

3 methodological concerns exist with the study. The first is that the method of concealing allocation to the intervention groups is not clear. Second, despite a high follow up rate, an intention to treat analysis was not carried out. Third, the method used to define atopic eczema was non-specific (eg, simple irritant contact dermatitis could have been included), ${ }^{1}$ and other validated definitions could have been used. These methodological concerns are unlikely to invalidate the study, but we do not know for sure unless we have more information or the study findings are replicated.

With respect to changing practice, 2 points are noteworthy. First, the results should not be generalised to those without a positive family history of atopy. The lack of change in atopic markers suggests that a study in non-atopic families might be worthwhile. Second, it remains to be seen whether the initial benefit in the intervention group is a sustained one, as opposed to merely delaying the onset of symptoms as other prevention studies have found. ${ }^{2}$ Long term data are also awaited on asthma, hay fever, and safety risk.

So, would I recommend probiotics to the families with atopic eczema that I care for? Not yet. Although this is an exciting study, history teaches me to be cautious about changing my practice on the basis of 1 moderately sized randomised controlled trial, the results of which are entirely dependent on 1 assessor. Additional studies are needed to confirm the findings, and longer follow up is needed to see whether the benefits are sustained.

Hywel C Williams, MSc, $\mathrm{PhD}$ Centre of Evidence-Based Dermatology Queen's Medical Centre Nottingham, UK

1 Williams HC, Burney PG, Pembroke AC, Hay RJ. The U.K. Working Party's Diagnostic Criteria for Atopic Dermatitis. III. Independent hospital validation. Br J Dermatol 1994; 131:406-16.

2 Mar A, Marks R. Prevention of atopic dermatitis. In: Williams $\mathrm{HC}$, ed. Atopic dermatitis: the epidemiology, causes and prevention of atopic eczema. New York: Cambridge University Press, 2000:205-20 